\title{
Uso de sustituto dérmico en niños con secuelas de quemaduras. Tres casos clínicos
}

\author{
M. BEATRIZ QUEZADA K. ${ }^{1}$, RICARDO AYALA M. ${ }^{1}$, VERÓNICA YÁÑEZ V. ${ }^{1}$ \\ 1. Corporación de Ayuda al Niño Quemado COANIQUEM. Centro de Rehabilitación Santiago.
}

\begin{abstract}
Dermis cutaneous substitute for burn sequelae. 3 case-reports.

Background: In COANIQUEM, burn sequelae ambulatory reconstructive surgeries in pediatric patients are performed during their rehabilitation treatments. One of these surgeries is the traditional technique of postburn contracture release and split-thickness skin grafting. A new alternative has been the appearance of a dermis substitute (Integra, Dermal Regeneration Template). There is no previous experience in Chile with this artificial skin applied in children with burn sequelae. Objective: Evaluate the ambulatory utilization of this dermis substitute in pediatric patients with burn sequelae and their evolution during two years. Case-reports: Surgeries carried out during 2004 in three patients 9 to 13 years-old, with upper extremity esthetic - functional sequelae. 24 months after surgery, a life- quality test (BSHS) and a rehabilitation protocol were applied. Results: There were no surgical complications in the three patients; the grafting attachment percentage varied between 75 and $100 \%$. All of them needed compression therapy for 13 months. The elasticity and folding were similar to normal skin two years after evolution, with absence of fibrosis and graft contraction. BSHS was favourable. One case presented mild infection. Conclusion: Dermis cutaneous substitute Integra constitutes a good treatment alternative and its ambulatory use is possible without surgical complications.

(Key words: pediatric patient, burn sequelae, Integra ${ }^{\circledR}$, ambulatory surgery).

Rev Chil Pediatr 2009; 80 (2): 150-156
\end{abstract}

\section{RESUMEN}

Introducción: En COANIQUEM se realizan cirugías reconstructivas de secuelas de quemaduras en forma ambulatoria, en pacientes pediátricos durante su tratamiento de rehabilitación. Una de estas cirugías es la técnica tradicional del desbridamiento más injerto dermo-epidérmico. Una nueva alternativa ha sido la aparición del sustituto dérmico Integra (Dermal Regeneration Template). En Chile, no existe experiencia con este sustituto dérmico aplicado en forma ambulatoria en niños con secuelas de quemaduras. Objetivo: evaluar la utilización de un sustituto dérmico en forma ambulatoria, en pacientes pediátricos con secuelas de quemadura y su evolución a dos años plazo. Casos clínicos: cirugías realizadas durante 2004 en tres

Trabajo recibido el 20 de noviembre de 2007, devuelto para corregir el 15 de abril de 2008, segunda versión el 03 de julio de 2008, aceptado para publicación el 18 de diciembre de 2008.

Se agradece a Promedón-Chile, por donación de plancha de Integra ${ }^{\circ} 20$ por $25 \mathrm{~cm}$.

Correspondencia a:

Dra. María Beatriz Quezada Kerr

E-mail: beatrizquezadakerr@yahoo.com. 
pacientes de 9 a 13 años de edad, con secuelas estético-funcionales de extremidad superior. Se aplicó protocolo de evaluación de rehabilitación a 24 meses y administración de test de calidad de vida (BSHS). Resultados: No hubo complicaciones quirúrgicas en los tres pacientes; el porcentaje de prendimiento varió entre 75 y $100 \%$. Todos requirieron presoterapia por 13 meses. La elasticidad y plegabilidad fue similar a la piel normal a los dos años de evolución, con ausencia de fibrosis y contracción del injerto. BSHS resultó favorable. Hubo presencia de infección leve en un caso. Conclusión: El sustituto dérmico Integra ${ }^{\circledR}$ es una buena alternativa de tratamiento y es posible su uso en forma ambulatoria sin mayores complicaciones quirúrgicas.

(Palabras clave: paciente pediátrico, secuela de quemadura, sustituto dérmico-integra ${ }^{\circledR}$, cirugía ambulatoria). Rev Chil Pediatr 2009; 80 (2): 150-156

\section{Introducción}

Las quemaduras constituyen un problema de salud pública en nuestro país por su prevalencia e impacto en la capacidad laboral de las personas, su calidad de vida y autoestima.

En COANIQUEM anualmente se atienden 7000 niños por quemaduras, de éstos el $20 \%$ requiere de rehabilitación por secuelas estético-funcionales. En el 32\% de estos casos es necesario realizar cirugías por su compromiso funcional, especialmente en localizaciones como extremidades superiores e inferiores. Las cirugías se realizan en forma ambulatoria basadas en el modelo quirúrgico de nuestro centro con 20 años de experiencia.

El desbridamiento más injerto es una de las técnicas quirúrgicas utilizadas con el objetivo de disminuir la tensión que genera la zona cicatricial.

Integra (Dermal Regeneration Template) es un sustituto dérmico bilaminar compuesto por una capa de colágeno de bovino y glicosaminoglicanos de tiburón y una capa de silicona. Esta matriz permite un proceso de cicatrización ordenado, formando una neodermis, con lo que se logra un resultado estético y funcional semejante a la piel normal.

Este procedimiento quirúrgico consta de dos cirugías: primero desbridamiento más colocación de sustituto dérmico y segundo retiro de la capa de silicona más la colocación de un injerto. Entre ambas cirugías, se requiere de atención de enfermería durante el período de formación de la neodermis que se extiende entre 14 y 21 días.

El objetivo de este trabajo fue evaluar la utilización de sustituto dérmico Integra en forma ambulatoria en tres pacientes pediátricos con secuelas de quemaduras y su evolución a dos años.

\section{Casos clínicos}

Se desarrolló un proyecto para el uso de una plancha 20 × $25 \mathrm{~cm}$ de Integra en el Centro de Rehabilitación-Santiago de COANIQUEM año 2004, que fue dividida con técnica estéril en láminas individuales proporcionales al tamaño de la zona cruenta y su posterior uso. La lámina fue cortada de acuerdo al tamaño requerido en una mesa separada del campo quirúrgico, cumpliéndose rigurosamente con los protocolos establecidos por el comité de IHH para evitar cualquier contaminación. La plancha no utilizada se guardó en un frasco con alcohol isopropílico según las especificaciones del fabricante para ser utilizada al día siguiente en las otras cirugías. Idealmente debe utilizarse una plancha para cada paciente.

Se seleccionaron tres pacientes con secuelas de quemaduras localizadas en extremidad superior, con compromiso funcional y estético, mayores de 8 años. Todos ingresaron a un protocolo prospectivo con evaluación interdisciplinario (médico fisiatra, cirujano y kinesióloga) que consideraba manejo en fase aguda, cirugías previas de secuelas, rehabilitación, adhesión a tratamiento, evaluación de la cicatriz, goniometría y actividades de la vida diaria. Se aplicó test de calidad de vida (BSHS) en el preoperatorio y a los 12 meses. El BSHS es un instrumento específico de medición de calidad de vida en pacientes quemados que consta de 37 preguntas agrupadas en 8 dominios que reflejan independencia funcional en actividades domésticas y escolares, funcionalidad manual, 
molestias o dificultades por el tratamiento, molestias por el calor o sol, relación con la familia, imagen propia y ánimo ${ }^{1}$.

Los niños permanecieron en la residencia de acogida para pacientes y padres que se encuentra en COANIQUEM durante el tiempo necesario para lograr la formación de neodermis.

Caso clínico 1: Paciente de Los Ángeles, 8 años, con secuela de quemadura en extremidad superior izquierda y compromiso funcional (déficit de $20^{\circ}$ de extensión) secundario a la retracción de queloide localizado en pliegue del codo.

Primera cirugía, 8 de noviembre de 2004, sin incidentes, desbridamiento hasta lecho sano, hemostasia rigurosa. Se colocó una lámina de 14 por $18 \mathrm{~cm}$. de Integra. Se utilizó profilaxis antibiótica con cefazolina una dosis endovenosa. Fijación de lámina con corchetes, malla elástica estéril directo sobre Integra, gasas empapadas con nitrato de plata. Vendaje y valva de yeso con codo en extensión.

Atención de enfermería, se realizaron instilaciones con nitrato de plata sobre gasas, 2 veces al día durante la primera semana, 1 vez al día la segunda y cada 48 horas la tercera semana.

Revisión médica postoperatoria periódica, evaluando presencia de hematomas, bulas, signos de infección o desprendimiento precoz de silicona. Se cambia valva de yeso por valva de termoplástico.

A los 16 días de evolución, después de iniciar movilización pasiva en box por kinesiólogo, apareció secreción purulenta escasa en relación a un corchete.

Segunda cirugía, 29 noviembre de 2004, se visualizó Integra de un color anaranjado amarillo homogéneo y liso, lo que nos indicó que la neodermis estaba formada. Se retiró silicona con facilidad, quedando un lecho apto que se cubrió con injerto fino dermo-epidérmico, tomado de cara lateral del muslo derecho. Vendaje tradicional, valva de termoplástico en extensión.

En la zona dadora se utilizó alginato de calcio más apósito adhesivo plástico.

Evolución postoperatoria 4to día: Injerto prendido en un $75 \%$; se tomó cultivo, encon- trándose cocos gram positivo, polimorfos nucleares escasos y desarrollo de Staphylococcus aureus, indicándose Flucloxacilina oral y Mupirocina local.

La zona cruenta residual se cubrió posteriormente con injerto, cicatrizando totalmente a los 44 días de la segunda cirugía. En los primeros controles postoperatorios se esperó una mejor evolución de las zonas dudosas de prendimiento sobre Integra, en el futuro no es necesario esperar más de 7 días para definir la necesidad de un nuevo injerto, lo que proporcionará un mejor resultado final.

Rehabilitación, se inició tratamiento compresivo del injerto sin problemas a los 23 días postoperatorio y, a los 45 días los ejercicios activos de flexo extensión y prono supinación.

A los 64 días se observó injerto de buen aspecto, flexible, bordes un poco solevantados y con mayor actividad, logrando flexión completa del codo. Llamó la atención al flectar el codo, la formación de un pliegue grueso de piel que correspondía al grosor de la lámina utilizada. Regresó a Los Ángeles, con indicaciones de presoterapia continua, ejercicios activos para mantener rangos articulares y masoterapia.

A los 5 meses desarrolló cicatriz queloídea en los bordes de la zona injertada, la zona central mostró rápidos signos de maduración con buena plegabilidad y elasticidad.

La presoterapia se mantuvo por 24 meses, debido a la presencia de los bordes queloídeos en los bordes de Integra; con piel sana no hubo mayor hipertrofia en la zona donde se perdió el injerto

A los 2 años de evolución, la paciente y su familia, se mostraron satisfechos con el resultado obtenido. Se realizaron pruebas funcionales comprobándose rango completo de extensión, sin retracción en la zona injertada con Integra, pero persistía borde queloídeo hipertrófico en regresión. Realiza vida normal y es independiente en sus actividades de vida diaria.

Caso clínico 2: Paciente de Linares, 9 años, con secuela quemadura en extremidad superior izquierda (figura 1).

Primera cirugía, 9 noviembre de 2004 se utilizó la misma técnica quirúrgica, colocándose una lámina de Integra de 9 por $15 \mathrm{~cm}$ (figura 2). 


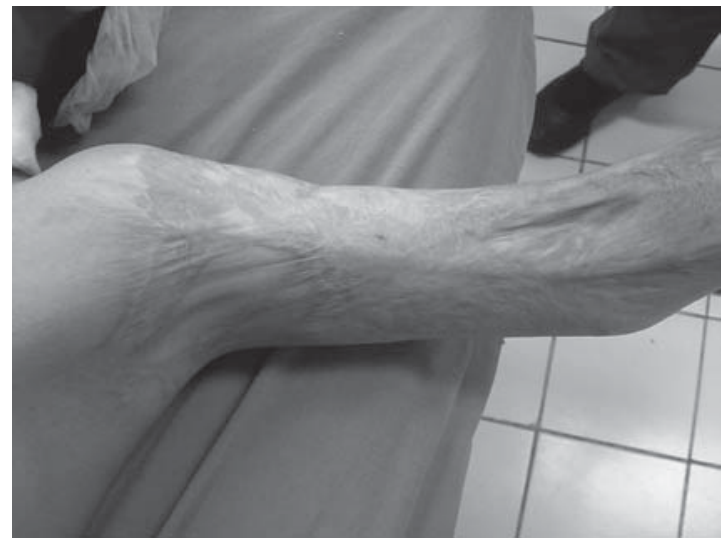

Figura 1. Caso clínico 2. Preoperatorio.

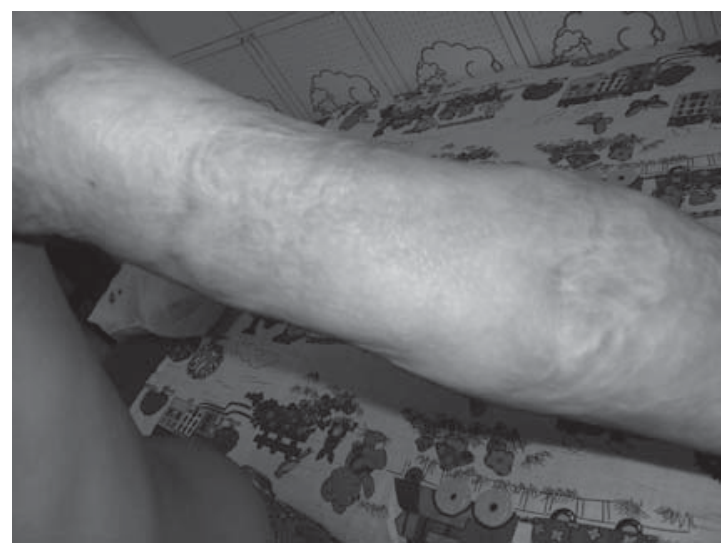

Figura 3. Evolución a los 18 meses.

Se realizó la misma atención de enfermería que en caso 1. A los 18 días se evidenció tejido granulatorio y separación de la capa de silicona, en una pequeña zona del borde, sin signos de infección.

Segunda cirugía, 29 noviembre de 2004, se visualizó neodermis formada que se cubrió con injerto fino tomado de muslo. El grosor del injerto no fue uniforme, quedando en el borde superior el injerto más grueso, que evolucionó con pérdida de éste requiriendo ser reinjertado en pabellón a los 36 días. Injerto pequeño tomado de antebrazo, evolucionó en forma satisfactoria con $100 \%$ de prendimiento. En los primeros controles postoperatorios se esperó una mejor evolución al igual que en el primer caso, idealmente la decisión de reinjertar debe ser tomada más precozmente.

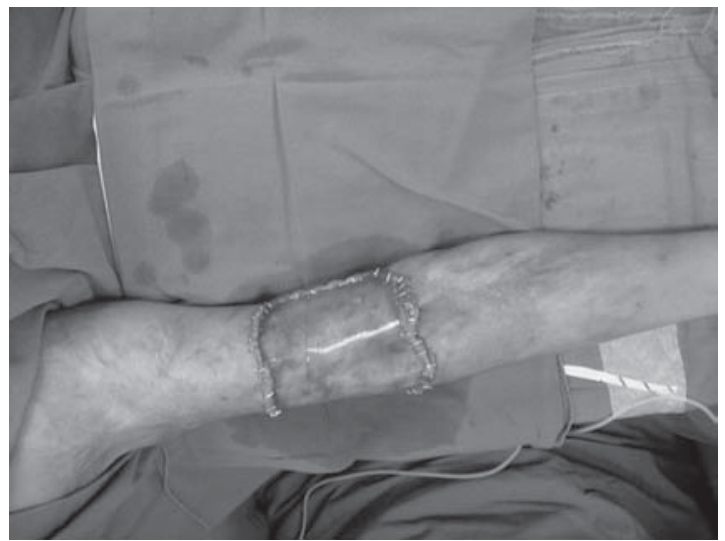

Figura 2. Sustituto dérmico recién colocado en la zona cruenta.

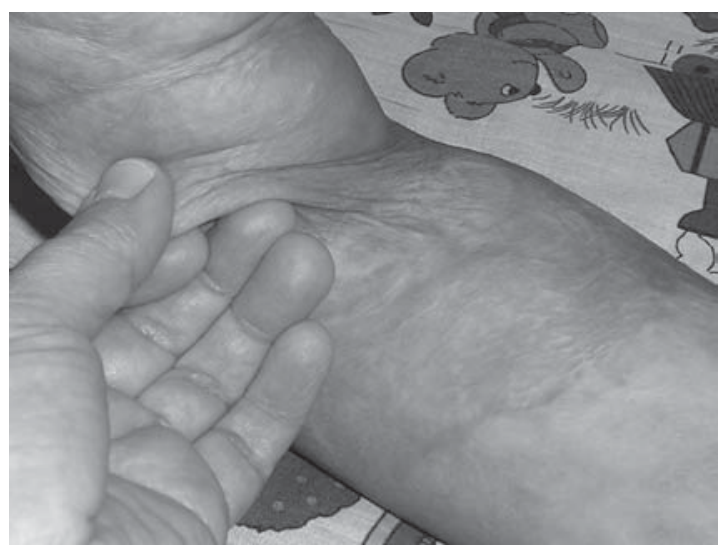

Figura 4. Evolución a los 18 meses, plegabilidad y elasticidad similar a la piel normal.

Rehabilitación, se inició tratamiento compresivo a los 7 días post reinjerto, y a los 15 días, ejercicios activos de flexo extensión, prono supinación, fortalecimiento muscular y masoterapia. Regresó a Linares a los 64 días.

A los 12 meses se visualizaron signos de maduración con buena plegabilidad y elasticidad de la zona injertada, se retiró la presoterapia a los 13 meses (figuras 3 y 4). A los 12 meses la zona injertada estaba madura, con mejor calidad que injerto dermoepidérmico (DE) o injerto piel total en cuanto a plegabilidad y elasticidad objetivada con pie de metro.

El paciente creció en este período, la zona injertada aumentó su tamaño en longitud $1 \mathrm{~cm}$ y $0,5 \mathrm{~cm}$ de ancho.

A los dos años, el BSHS aplicado al paciente y su familia, es muy positivo respecto evolu- 
ción, no refiere molestias y estaría dispuesto a utilizar este sustituto dérmico nuevamente si fuese necesario.

Caso clínico 3: Paciente de Santiago, 13 años 9 meses, con secuela localizada en antebrazo izquierdo.

Primera cirugía, 10 noviembre de 2004, se realizó un desbridamiento en región del antebrazo, se colocó lámina de Integra 10 por $10 \mathrm{~cm}$.

Atención de enfermería, se realizaron las instilaciones y revisiones médicas periódicas según protocolo, con cambio de valva de yeso a termoplástico a los 8 días de evolución, lo que facilita su rápido retiro, instalación y comodidad por ser más liviano. La valva de yeso sólo se utiliza en el postoperatorio inmediato. A los 13 días comenzó con ejercicios pasivos de movilización.

Segunda cirugía, 30 noviembre de 2004. Se visualizó neodermis formada cubriéndose con injerto finísimo tomado de cara lateral de muslo. Otra zona donante puede ser cuero cabelludo, por la edad de los pacientes no aceptaron utilizar esa zona; en nuestra experiencia al utilizar alginato de calcio en las zonas dadoras, la secuela estética es prácticamente nula. Evolución postoperatoria, injerto $100 \%$ prendido. Inició terapia compresiva a los 10 días de evolución, comenzando con ejercicios activos para aumentar rangos articulares y a los 2 meses se indicó masoterapia.

Rehabilitación, A los 12 meses se visualizaron signos de maduración con buena plegabilidad y elasticidad de la zona injertada; se retira la presoterapia a los 13 meses, continuando con pauta de ejercicios. Se produjo una mínima retracción de la zona injertada (menos de $0,5 \mathrm{~cm})$.

A los 24 meses de evolución, presentó plegabilidad y elasticidad similar piel normal con rangos articulares completos. Según BSHS, paciente muy contenta con su resultado estético y funcional.

\section{Resultados}

En esta serie de casos no hubo complicaciones quirúrgicas mayores. Sólo se presentó una complicación infecciosa, purulenta en los bor- des en relación a los corchetes. El porcentaje de prendimiento en promedio fue superior al $85 \%$, concordante con lo encontrado en literatura $^{2}$.

Se inició presoterapia en promedio a los 10 días postinjerto a pesar de la presencia de algunas zonas cruentas. La utilización de órtesis de termoplástico comenzó durante la primera semana para facilitar la comodidad del paciente y se mantuvo hasta el inicio de la presoterapia. A los 14 días se iniciaron las movilizaciones y a las seis semanas ejercicios activos. La masoterapia comenzó a los 2 meses postinjerto. La maduración del injerto fue a los 12 meses y el retiro de la presoterapia a los 13 meses en dos pacientes y la tercera se mantuvo por 24 meses por la presencia de queloide en el borde de la cicatriz.

La utilización de sistemas compresivos es menos complejo y más corto, estimándose una disminución en el tiempo de maduración de la cicatriz aproximadamente entre 10 y $25 \%$, respecto al resto de pacientes Coaniquem en quienes no se utilizó Integra, con excepción de la primera paciente, quien desarrolló nuevamente queloide en el borde cicatricial del sustituto dérmico.

La calidad de la cicatriz, elasticidad y plegabilidad fue excelente, muy similar a la piel normal, incluso se evidenció un aumento de longitud de la lámina, sin desarrollar tensión, brida ni limitación articular, en el menor que se encontraba en plena etapa de crecimiento. En el caso de injertos DE sin sustituto dérmico también puede observarse este crecimiento.

A los dos años, existía aún hipercromía de la zona injertada similar al injerto tradicional. El aspecto estético correspondía a un injerto de muy buena calidad.

Al comparar los resultados de la aplicación del BSHS (en momento inicial y un año después), los principales cambios se refieren a la mejoría del estado emocional y la percepción estética de la cicatriz. Se aprecia buen nivel de calidad de vida.

Los pacientes se encontraban contentos y muy satisfechos con el resultado obtenido, especialmente desde el punto de vista funcional. Volverían a intentar el mismo procedimiento si fuese necesario. 


\section{Discusión}

El sustituto dérmico Integra fue utilizado por Yannas y Burke en $1981^{2}$, inicialmente en pacientes con grandes extensiones de quemaduras que no tenían suficientes zonas dadoras. Posteriormente comienza su utilización en pacientes con secuelas de quemaduras ${ }^{3}$.

Durante la formación de neodermis, se produce regeneración tisular en forma ordenada que puede ser demostrada histológicamente en cuatro etapas: imbibición, migración de fibroblastos, neovascularización y remodelación ${ }^{4}$. Las fibras de colágeno del paciente se disponen de manera similar a la histología normal; al mismo tiempo, las fibras de colágeno de bovino y glicosaminoglicanos son fagocitadas. Este proceso de neovascularización dura entre 14-21 días, durante los cuales se produce un cambio gradual de coloración que indica el momento adecuado para reemplazar la capa superior de silicona por un injerto finísimo del mismo paciente.

En la primera semana al existir esta membrana acelular se produce mayor riesgo de infección, siendo ésta una de las complicaciones más frecuentes y temidas en el uso de sustitutos dérmicos. Otra complicación esperable en las primeras horas es la formación de coágulos o hematomas que puedan impedir la adherencia íntima al lecho receptor con lo cual no puede formarse la neodermis.

Para prevenir estas complicaciones es muy importante la hemostasia rigurosa. Mantener una adecuada inmovilización y disminuir todos los riesgos de infección.

Uno de los desafíos de este trabajo era asegurar todas las condiciones de inmovilización, controles y cuidados de enfermería en pacientes pediátricos en forma ambulatoria, que se logró con la estadía de los pacientes en la casa de acogida.

Al comparar el resultado obtenido con Integra con los desbridamientos más injertos dérmopidérmicos tradicionales, a los dos años de evolución es posible encontrar similitudes, como crecimiento de los injertos junto con el niño y plegabilidad similar a la piel normal. La importancia de este estudio es conocer la aplicación de este sustituto dérmico en niños en forma ambulatoria y su aplicación en secuelas de quemaduras.

En estudios publicados, Integra constituye una excelente alternativa en el tratamiento de secuelas de quemaduras, el proceso de rehabilitación es más corto que con las técnicas tradicionales de desbridamiento más injerto ${ }^{5}$. La calidad de la cicatriz, plegabilidad, elasticidad y su resultado estético funcional también es superior, al igual que el grado de satisfacción del paciente al compararlo con otras zonas injertadas $^{6}$.

En experiencias en niños además existe una buena evolución con el crecimiento, en la mayoría de los casos se produce un crecimiento normal de la zona con Integra ${ }^{7}$.

Otra de las ventajas de la utilización del sustituto es la posibilidad de extirpar cicatrices extensas o efectuar amplios desbridamientos a pesar que el sitio donante no sea del mismo tamaño, ya que en algunos casos es posible colocar injerto en malla con igual resultado estético posterior. Además al utilizarse un injerto finísimo se obtendrá una menor secuela en la nueva zona dadora ${ }^{8}$.

\section{Conclusiones}

El costo elevado de este sustituto dérmico, la necesidad de dos cirugías y la atención prolongada de enfermería, constituyen desventajas importantes para su utilización.

El excelente resultado estético-funcional, con plegabilidad y elasticidad similar a la piel normal, ausencia de fibrosis y contracción de los injertos, a dos años plazo, justifican su uso; Integra constituye una alternativa real de tratamiento para algunos pacientes pediátricos con secuelas de quemaduras y es posible su uso en forma ambulatoria sin mayores complicaciones en nuestro Centro de Rehabilitación.

\section{Referencias}

1.- Kindal M, Andersson G, Fulg-Meyer AR, Lannerstam $K$, Gerdin B: Development of a brief vertion of the burn specific health scale. J Trauma 2001; 51: 740-6.

2.- Burke JF, Llanas JV, Quinby WC, Bondoc CC, Jung $W K$ : Successful use a physiologically acceptable artifi- 
cial skin in the treatment of extensive burn injury. Ann Surg 1981; 194: 413.

3.- Frame JD, Still J, Lakhel-LeCoadou A, et al: Use of dermal regeneration template in contracture: a multicenter evaluation. Plast Reconstr Surg 2004; 113 (5): 1330-8.

4.- Moiemen NS, Staiano JJ, Ojeh NO, Thway Y, Frame $J D$ : Reconstructive surgery with a dermal regeneration template: clinical and histologic study. Plast Reconstr Surg 2001; 108 (1): 93-103.

5.- Martínez L, Roz Z, López-Gutiérrez JC, et al: Integra Artificial dermis in pediatric reconstructive surgery.
Cir Pediatr 2002; (3): 97-100.

6. - Groos N, Guillot M, Zilliox R, Braye FM: Use of an artificial dermis (Integra) for the reconstruction of extensive burn scars in children. About 22 grafts. Eur J Pediatr Surg 2005; 15 (3): 187-92.

7.- Jeng JC, Fidler PE, Sokoloich JC, et al: Seven years' experience with Integra as a reconstructive tool. J Burn Care Res 2007; 28 (1): 120-6.

8:- Trong-Duo Chou, Shao-Liang Chen, Tz-Wen Lee, et al: Reconstruction of burn scar of the Upper Extremities wih Artificial Skin. Plast Reconst Surgery 2001; 108 (2): 378-84. 